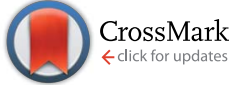

Cite this: RSC Adv., 2017, 7, 12876
Received 20th December 2016 Accepted 6th February 2017

DOI: $10.1039 /$ c6ra28495g

rsc.li/rsc-advances

\section{One pot synthesis of amine-functionalized and angular-shaped superparamagnetic iron oxide nanoparticles for MR/fluorescence bimodal imaging application $\dagger$}

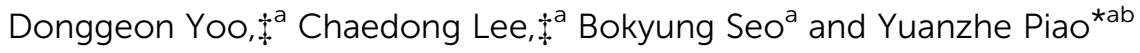

Herein, we report the simple preparation of water dispersible angular-shaped amine-functionalized superparamagnetic iron oxide nanoparticles (A-SPIONs). A-SPIONs were synthesized by heating iron(III) acetylacetonate in a mixture of solvents containing polyethyleneglycol (PEG) and branched polyethyleneimine ( $b-P E I)$ under vigorous stirring. Both PEG and b-PEI provided high water dispersibility by competitively surface coating the A-SPIONs. In addition, b-PEI controlled the overall morphology of the A-SPIONs, producing polyhedral nanocrystals in combination with the added halide ions. Due to the amine functional group from b-PEI, the A-SPIONs are proven to have both a positively charged surface $(+29.1 \mathrm{mV})$ and active sites, which enable facile functionalization. Using A-SPIONs of $9.42 \pm 2.93 \mathrm{~nm}$ (TEM observation), a high saturation magnetization value of $75.61 \mathrm{emu}^{-1}$ was obtained using a superconducting quantum interface device (SQUID). The A-SPIONs sustained a stable dispersion in aqueous media with various $\mathrm{pH}$, and their hydrodynamic size was about $13.97 \mathrm{~nm}$ in $0.10 \mathrm{M} \mathrm{NaCl}$ solution. Through the MTT assay, the A-SPIONs were proven to have negligible cellular toxicity in SKOV3, U87-MG, and U251 cell lines. The angular-shaped iron oxide nanoparticles also exhibited high relaxivity for magnetic resonance imaging (MRI), which originated from their high magnetization. Cyanine 5.5 dye-functionalized A-SPIONs (Cy 5.5@A-SPIONs) were prepared and serial experiments were conducted to investigate their fluorescence imaging applications. According to the results, the A-SPIONs are expected to have potential applications in bimodal imaging.

\section{Introduction}

With the advancement of nanotechnology, nanosized materials have been investigated as novel bioimaging probes and theranostic agents in various biomedical research areas due to their many unique properties. ${ }^{1}$ Especially, superparamagnetic iron oxide nanoparticles (SPIONs) have been widely researched for the development of multimodal bioimaging nanoprobes by merging with other functional components such as fluorescent dyes, ${ }^{2}$ radioisotopes, ${ }^{3}$ and anti-cancer drugs. ${ }^{4-7}$

There have been many efforts in the synthesis of iron oxide nanoparticles (IONPs) via various methods such as co-precipitation, $^{\mathbf{8 - 1 0}}$ thermal decomposition, ${ }^{\mathbf{1 1 - 1 3}}$ hydrothermal, ${ }^{\mathbf{1 4 , 1 5}}$

\footnotetext{
aProgram in Nano Science and Technology, Graduate School of Convergence Science and Technology, Seoul National University, 145 Gwanggyo-ro, Yeongtong-gu, Suwon-si, Gyeonggi-do, 443-270, South Korea. E-mail: parkat9@snu.ac.kr; Fax: +8231-8889148; Tel: +82-31-8889141

${ }^{b}$ Advanced Institutes of Convergence Technology, 145 Gwanggyo-ro, Yeongtong-gu, Suwon-si, Gyeonggi-do, 443-270, South Korea

$\dagger$ Electronic supplementary information (ESI) available. See DOI: 10.1039/c6ra28495g

\$ These authors contributed equally.
}

solvothermal, ${ }^{16,17}$ and polyol synthesis. ${ }^{18,19}$ Among these, polyol synthesis was discovered to overcome some problems such as low crystallinity, ${ }^{20}$ post-synthetic processes for water dispersibility, ${ }^{21-23}$ and lack of morphological uniformity. ${ }^{24}$ Since a biocompatible polymer, polyethyleneglycol (PEG), is used as both a solvent and a reducing agent in the polyol process, the synthesized nanoparticles are highly water dispersible and biocompatible without further functionalization steps such as ligand exchange and phase transfer. ${ }^{19}$

In biological applications, one of the important issues in nanoparticle synthesis is chemical modification on the surfaces. Click chemistry, ${ }^{25,26}$ amide-bond formation, ${ }^{27}$ and disulfide interactions ${ }^{28,29}$ are common approaches in the surface modification. However, these post syntheses require additional reaction time, considerable number of reagents, and further purification steps.

The physicochemical properties of the nanoparticles are highly dependent on their size and shape. Significant amount of research has been conducted on the preparation of IONPs with various shapes by optimizing the heating rate, reaction temperature, and aging time. ${ }^{11-13}$ For example, Park et al. ${ }^{30}$ achieved nanoworm-shaped IONPs to enhance the blood 
circulation time and Guardia et $a l .{ }^{31}$ synthesized cubic-shaped IONPs to improve the hyperthermia effect. Moreover, Zhen et $a .^{32}$ compared the magnetic properties of spherical and cubic-shaped IONPs that were similar in size and concluded that the cubic IONP showed an enhanced magnetization value.

Furthermore, the shape of iron oxide nanoparticles can be adjusted by adding halide ions or metal-chelating polymers. Zhao et $a .^{33}$ reported the synthesis of octapod IONPs in the presence of sodium chloride and achieved high-performance $T_{2}$ contrast from in vivo MR imaging, whereas $\mathrm{Xu}$ et al. ${ }^{34}$ synthesized iron oxide using halide ions in organic solvent.

Polyethyleneimine (PEI) is a polymer with repeated amine groups and two carbon aliphatic $\mathrm{CH}_{2} \mathrm{CH}_{2}$ spacers. Due to the abundance of amine functional groups, PEI has been used to control the surface charge $\mathrm{e}^{35}$ and to introduce other functional groups $^{36}$ such as carboxylic acid groups via amide-bond formation. Furthermore, because of its positive charge originating from the amine functional group, PEI has been used in the layer-by-layer (LBL) process to attach the oppositely charged moieties via physical interaction. ${ }^{35}$ Moreover, PEI also has been used as an attachment promoter in cultured cells because PEI can facilitate weak anchoring of the cells with a negatively charged outer surface to stick to the cell plate. ${ }^{37}$ In addition, PEI also has been employed as a chelating agent with the ability to complex heavy metal ions such as zirconium and zinc. ${ }^{38}$ Recently, Yu et al. reported the novel synthesis of metal nanoparticles by controlling the ratio of the metal precursor and PEI, producing certain nanoparticles with various shapes such as cubic, triangular, and so on. ${ }^{39,40}$

In this study, we proposed the facile direct synthesis of angular-shaped and amine-functionalized super-paramagnetic iron oxide nanoparticles (A-SPIONs). A-SPIONs were aminefunctionalized via a facile one-step procedure, which is an advantage in the engineering aspect for their biomedical applications. Furthermore, coincident passivation of halide ions and b-PEI resulted in polyhedral nanocrystals with superior magnetic property, which is highly relevant to their contrast enhancement in MR imaging. Surface-anchored PEG and b-PEI chains enabled stable dispersions in various $\mathrm{pH}$ and diluted saline solutions. The cellular toxicity of A-SPIONs was investigated using three types of cell lines and their uptake study was also conducted via confocal microscopy. A near-infrared dye (Cyanine 5.5) was labelled to avoid any optical interference with the A-SPIONs. Based on these results, we believe that A-SPIONs are promising candidates for multipotent platforms in biomedical applications.

\section{Experimental}

\section{Materials}

Ferric nitrate nonahydrate $\left(\mathrm{Fe}\left(\mathrm{NO}_{3}\right)_{3} \cdot 9 \mathrm{H}_{2} \mathrm{O}\right)$, polyethyleneglycol 600 (PEG), diethyl ether, potassium bromide ( $\mathrm{KBr}$ ), and sodium chloride $(\mathrm{NaCl})$ were purchased from Samchun Chemical. Iron(III) acetylacetonate (>99\%) was purchased from STREMCHEM. Branched polyethyleneimine (b-PEI) and phosphate buffered saline (PBS) tablets of $\mathrm{pH} 7.4$ were acquired from Sigma Aldrich. Flamma 675 NHS ester was purchased from
BioActs (South Korea). Dimethyl sulfoxide (DMSO) was purchased from Junsei. Hoechst 33342 and WGA-1 were purchased from Thermo Fisher Scientific. All the reagents were used without further purification and aqueous solutions were prepared using high purity deionized water (18.2 M $\Omega$ ).

\section{Characterization}

Powder X-ray diffraction (XRD) patterns of the A-SPIONs were obtained using a Rigaku Dmax 2500 diffractometer with $\mathrm{Cu}-\mathrm{K} \alpha$ radiation $(\lambda=1.5406 \AA)$ at $40 \mathrm{kV}$ and $100 \mathrm{~mA}$. Transmission electron microscopy (TEM) images were obtained via a LIBRA 120 (Carl Zeiss) at an accelerating voltage of $120 \mathrm{kV}$ and high resolution TEM (HRTEM) images were obtained using a JEM3010 (JEOL) at an accelerating voltage of $300 \mathrm{kV}$. The hydrodynamic size distribution of the A-SPIONs dispersed in water was measured by a Zetasizer Nano ZS equipped with a He-Ne laser operating at $633 \mathrm{~nm}$ and a back-scattering detector at $173^{\circ}$ (Malvern). FT-IR spectra were obtained using a Nicolet iS10 in the attenuated total reflectance (ATR) mode (Thermo scientific Fisher corp.). Fluorescence excitation and emission spectra were obtained using a FluoroMate FS-2 (Scinco). Thermogravimetric analysis was carried out using a TGA/DSC 1 thermo gravimetric analyzer (Mettler-Tolledo). An inductively coupled plasma-emission spectrometer (Shimadzu, JP/ICPS-7500) was used to quantify the iron concentration of the A-SPIONs dispersed in water. In vitro fluorescence images were observed via confocal microscopy (A1-Rsi, Nikon). Magnetic measurement was conducted using a PPMS-14 (Quantum Design).

\section{Synthesis of the ultra-small PEGylated iron oxide nanoparticles}

Ultra-small PEGylated iron oxide nanoparticles (USPIOs) were prepared for comparison. The synthesis was carried out according to a previously reported procedure. ${ }^{19}$ Briefly, $0.5 \mathrm{mmol}$ of $\mathrm{Fe}\left(\mathrm{NO}_{3}\right)_{3} \cdot 9 \mathrm{H}_{2} \mathrm{O}$ was mixed with $10 \mathrm{mmol}$ of PEG. The resulting mixture was heated to $95{ }^{\circ} \mathrm{C}$ at a constant heating rate of $2.3{ }^{\circ} \mathrm{C} \mathrm{min}^{-1}$ and kept at this temperature for $30 \mathrm{~min}$. After this, the mixture was heated to $265^{\circ} \mathrm{C}$ at the heating rate of $5{ }^{\circ} \mathrm{C} \min ^{-1}$ and was kept at this temperature for $30 \mathrm{~min}$. The synthesis of PEGylated IONPs was carried out under very low pressure $(-76 \mathrm{~cm} \mathrm{Hg})$ to remove the generated impurities present in PEG using a Schlenk line. During the process, the initial transparent red-colored mixture changed to brown when the temperature was above $140{ }^{\circ} \mathrm{C}$. At the end of the reaction, a brownish black solution was obtained, indicating the formation of USPIO. Subsequently, the resulting product was cooled down to room temperature by removing the heating mantle. An ethanol-ether mixture was added to the resulting solution and the nanoparticles were separated by centrifugation with a relative centrifugal force (RCF) of 8000 .

\section{Synthesis of the angular-shaped and amine-functionalized super-paramagnetic iron oxide nanoparticles with different weight ratios of PEG and b-PEI}

The angular-shaped and amine-functionalized superparamagnetic iron oxide nanoparticles (A-SPIONs) were 
synthesized using a mixture of PEG and b-PEI with different weight ratios. A $0.5 \mathrm{mmol}$ of iron(III) acetylacetonate was added to $6 \mathrm{~g}$ of PEG and b-PEI mixtures with different weight ratios (PEG : b-PEI $=5: 1,1: 5$, and $0: 6$ ). The mixture was heated at $95{ }^{\circ} \mathrm{C}$ for 1 hour with low pressure $(-76 \mathrm{~cm} \mathrm{Hg})$ to eliminate $\mathrm{H}_{2} \mathrm{O}$ present in PEG and b-PEI. After the degassing step, the temperature was increased to $265{ }^{\circ} \mathrm{C}$ at the heating rate of $5{ }^{\circ} \mathrm{C}$ $\min ^{-1}$. During the process, the initial transparent red solution gradually changed to brown when the temperature was above $140{ }^{\circ} \mathrm{C}$. Subsequently, a brownish black solution was obtained at the end of the reaction. After the reaction, the resulting solution was cooled down to room temperature by removing the heating mantle. An ethanol-ether mixture was added to the resulting solution and the nanoparticles were separated by centrifugation with an RCF of 8000.

\section{Synthesis of the angular-shaped IONPs with halide ions ( $\mathrm{KBr}$ and $\mathrm{NaCl}$ )}

The A-SPIONs were synthesized using a mixture of PEG and bPEI (weight ratio, 5:1) in the presence of either potassium bromide or sodium chloride. Typically, $0.5 \mathrm{mmol}$ of iron(III) acetylacetonate precursor was dissolved in the PEG and b-PEI mixture and $1 \mathrm{mmol}$ of potassium bromide or sodium chloride was added to the mixture. The heating and washing steps were the same as abovementioned. Moreover, the added amount of $\mathrm{NaCl}$ was changed from 0 to $5 \mathrm{mmol}$ to study the morphological variations.

\section{Synthesis of Cy 5.5@A-SPIONs}

Cyanine 5.5 dye (Flamma 675-NHS ester) was used for the fluorescent labelling of the as-prepared A-SPIONs. Since this dye molecule has a single NHS end, as shown in Fig. $\mathbf{S} 7, \uparrow$ the amide bonding with the amine end of A-SPIONs was directly achieved. To prepare Cy 5.5@A-SPIONs, 2 mL of the as-synthesized ASPIONs solution was washed three times with a mixture of ethanol and ether by centrifugation and redispersed in $2 \mathrm{~mL}$ methanol. Then, $1 \mathrm{~mL}$ of $9.6 \times 10^{-4}$ M Cy 5.5 methanol solution was added and stirred at room temperature for 2 hours. After this, $8 \mathrm{~mL}$ of ethanol-ether mixture $(1: 3 \mathrm{v} / \mathrm{v})$ was added to the solution under vigorous stirring and the resulting Cy 5.5@ASPIONs were separated by centrifugation with an RCF of 8000 3 times to remove the unreacted Cy 5.5 dye. The obtained Cy 5.5@A-SPIONs were redispersed in $2 \mathrm{~mL}$ of DI water.

\section{Cellular viability test}

Three types of cell lines (SK-OV-3: human ovarian cancer, U-251: human glioblastoma astrocytoma, and U87-MG: human glioblastoma astrocytoma were provided from the Korean Cell Line Bank) were used to investigate the cellular toxicity of the asprepared nanoparticles. The cell lines were grown in RPMI-1640 and DMEM (Gibco-BRL) media solution supplemented with $10 \%$ heat-inactivated fetal calf serum (BSA) and 1\% penicillin/ streptomycin (Gibco). For the analysis of cellular viability, each cell line was cultured in a 12-well plate $\left(\sim 1.0 \times 10^{5}\right.$ cells per well $)$ with a certain concentration of A-SPIONs. After incubating for 3, 6, 24, and 48 hours, the supernatant media was discarded and washed with 0.01 M PBS (phosphate buffered saline) solution. Then, each well was filled with $1 \mathrm{~mL}$ MTT solution (3-(4,5dimethylthiazol-2-yl)-2,5-diphenyltetrazolium bromide, $0.5 \mathrm{mg}$ $\mathrm{mL}^{-1}$ ) and incubated for one hour. Again, the supernatant solution was eliminated and $500 \mu \mathrm{L}$ of DMSO was added to each well to break the cell membrane and dissolve the formazan crystals. Then, the absorbance value of each well was measured using a microplate reader ( $\mu$ Quant, BIO-TEK INSTRUMETNS, INC.). Each absorbance value was divided by that of the control experiment for relative comparison.

\section{In vitro cell imaging}

The RAW 264.7 cell line (macrophage from mouse, provided from the Korean Cell Line Bank) was grown in DMEM (GibcoBRL) media solution supplemented with $10 \%$ heat-inactivated fetal calf serum (BSA) and 1\% penicillin/streptomycin (Gibco). For high resolution confocal imaging, about $1 \times 10^{5}$ cells were cultured in a confocal dish (SPL life science, 100350). After 5 hours, the upper media solution was replaced with fresh media containing Cy 5.5-labelled A-SPIONs ( $40 \mu \mathrm{g}$ Fe per $\mathrm{mL}$ ) and was incubated for 12 hours. The cell nucleus and overall membrane were stained with Hoechst 33342 and WGA-1, respectively, and the images were obtained using an A1 Rsi confocal microscope $(\times 60)$.

\section{MR phantom test}

Phantom images were obtained using a 9.4 T/160 AS animal MRI system (Agilent Technologies, Santa Clara, CA, USA). The transverse relaxation time $T_{2}$ mapping was estimated using MEMS (multi echo multiple slice) sequences with a spin-echo readout. The sequence parameters were as follows: $\mathrm{TR}=3000$ $\mathrm{ms}, \mathrm{TE}=8.50 \mathrm{~ms}, \mathrm{NE}=16$, average $=1$, matrix size $=128 \times$ 128 , FOV (field of view) $=60.0 \times 60.0 \mathrm{~mm}^{2}$, slice thickness $=$ $2.0 \mathrm{~mm}$, and scan time $=6 \mathrm{~min} 30 \mathrm{~s}$.

\section{Results and discussion}

\section{Synthesis of angular-shaped superparamagnetic iron oxide nanoparticles (A-SPIONs)}

In a typical process (Scheme 1), the angular-shaped iron oxide nanoparticles were prepared via PEG-assisted polyol synthesis based on the previously reported method. ${ }^{19}$ During the polyol synthesis, the added b-PEI and $\mathrm{NaCl}$ attached on the surface of the iron oxide nanoparticles, facilitating the angular aspects and uniform size distribution. The anchored b-PEI structures generated numerous amine groups on the surface of the nanoparticles and enabled further functionalization to attach the Cy 5.5 dye on the surface of A-SPIONS, where we employed the commonly used amine-NHS ester reaction in methanol solvent.

The synthesis of A-SPIONs was conducted under the various reaction conditions to understand the surface-binding mechanism of b-PEI (Fig. 1). The amount of iron precursor was fixed (0.5 mmol) and only the weight ratio of PEG and b-PEI was changed as $0: 6,1: 5$, and $5: 1$. First, when there was no PEG during the synthesis ( $6 \mathrm{~g}$ of b-PEI only), iron oxide nanoparticles 


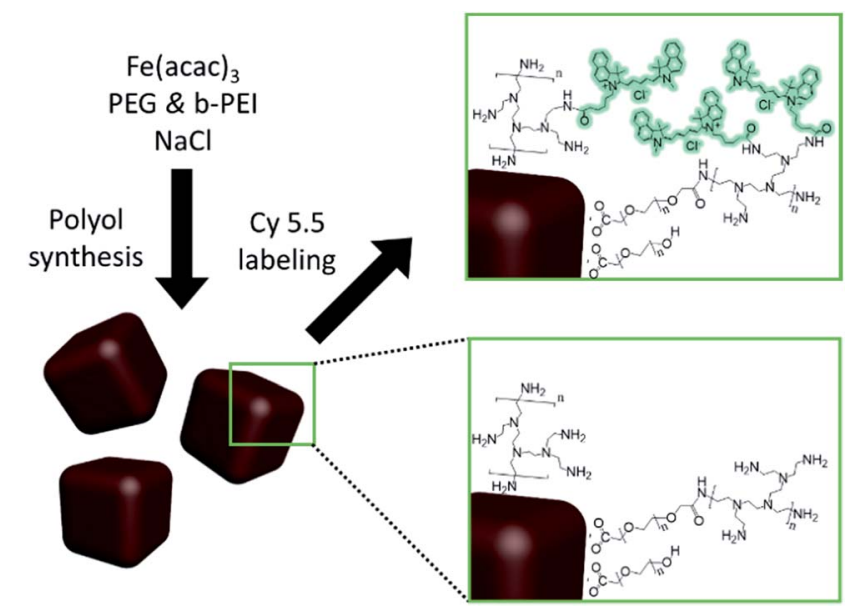

Scheme 1 Schematic for the synthesis of A-SPIONs and Cy 5.5@ASPIONs.
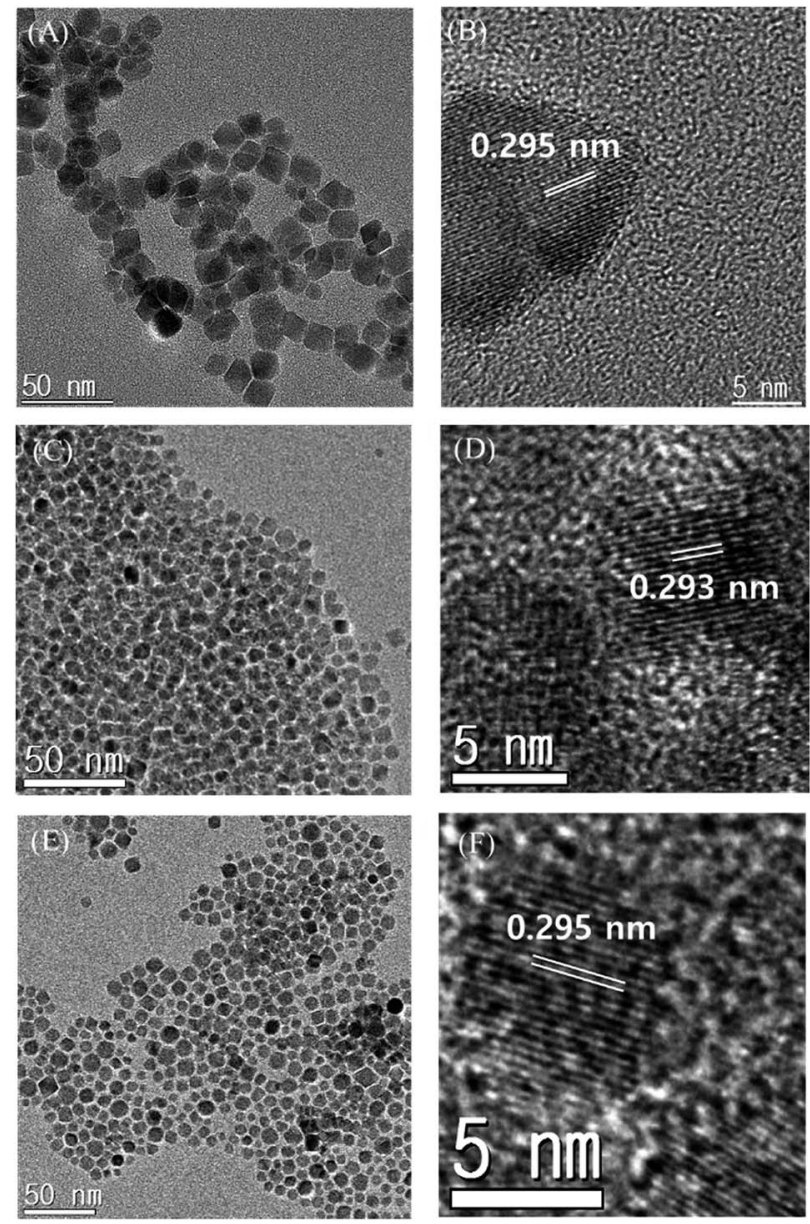

Fig. 1 TEM images of the synthesized nanoparticles prepared using various weight ratios of $P E G$ and $b-P E I, 0: 6$ ( $A$ and $B), 1: 5$ ( $C$ and $D)$, and $5: 1$ ( $E$ and $F$ ). Lattice distances (d-spacing) of the synthesized nanocrystals were measured from the magnified HR-TEM images ( $B, D$ and F). of $17.41 \pm 6.83 \mathrm{~nm}(\sigma=2.68)$ were obtained, which exhibit distinctive anisotropic shapes due to the amine-mediated surface binding of b-PEI (Fig. 1A). Second, in the case of the synthesis using the PEG and b-PEI solvent mixture with a weight ratio of $1: 5$, the size of the nanocrystals (Fig. 1C) was measured to be $7.70 \pm 2.62 \mathrm{~nm}(\sigma=1.20)$, which is smaller than that of the abovementioned crystals. Third, when the nanoparticles were synthesized in a solvent mixture of $5 \mathrm{~g}$ PEG $+1 \mathrm{~g}$ b-PEI, the size of the obtained nanoparticles (Fig. 1E) was measured to be 9.08 $\pm 2.70 \mathrm{~nm}(\sigma=2.21)$ and there was no big difference in the overall morphology of the second and third samples. As the content of PEG increased in the reaction solvent, the synthesized nanoparticles showed stable dispersion after the purification steps using ethanol and ether. Through HR-TEM, the lattice distance of the as-synthesized nanoparticles was measured to be $0.295 \mathrm{~nm}$, which is almost close to the reference data of the (220) planes of magnetite, $\mathrm{Fe}_{3} \mathrm{O}_{4}$ (Fig. 1B, D and F). ${ }^{41}$

As previously reported, halogens can facilitate the synthesis of cubic-shaped iron oxide nanoparticles by dissociating from the haloids and stabilizing the (100) facets of the nanocrystals. ${ }^{34}$ To enhance the angular morphology of the A-SPIONs, $1 \mathrm{mmol}$ of the halide ion source, such as sodium chloride or potassium bromide, was added to the reaction mixture with a 5 : 1 (5 g PEG $+1 \mathrm{~g}$ b-PEI) ratio (Fig. 2). From the TEM observation, it was observed that the A-SPIONs with $1 \mathrm{mmol}$ of $\mathrm{KBr}$ (Fig. 2A) showed polyhedral morphologies with a wide size distribution. Fig. 2B shows the size distribution of the randomly selected A-SPIONs with $1 \mathrm{mmol}$ of $\mathrm{KBr}(n=100)$, in which the mean size was calculated to be $13.49 \pm 10.53 \mathrm{~nm}(\sigma=5.4)$. Fig. $2 \mathrm{C}$ is the TEM image of the A-SPIONs with $1 \mathrm{mmol}$ of $\mathrm{NaCl}$.

Compared to the case of $\mathrm{KBr}$, relatively monodispersed iron oxide nanoparticles were obtained with $\mathrm{NaCl}$. Fig. 2D represents the size distribution of the randomly selected A-SPIONs with $1 \mathrm{mmol}$ of $\mathrm{NaCl}(n=100)$, in which the mean size was calculated to be $9.43 \pm 2.93 \mathrm{~nm}(\sigma=1.40)$. As a control experiment, we also synthesized iron oxide nanoparticles without b-PEI to confirm the influence of the added halide ions (Fig. S2A and $\mathrm{B} \dagger$ ). In the absence of the added halide ions, no prominent anisotropic aspects in the shape of A-SPIONs were observed. Moreover, the quantity of $\mathrm{NaCl}$ added was tuned from 0 to $5 \mathrm{mmol}$ to find the optimum conditions to prepare A-SPIONs. Through these experiments and thorough TEM observation, it was found that the concentration of chloride ions strongly influenced the shape of the nanoparticles. Using up to $3 \mathrm{mmol}$ of $\mathrm{NaCl}$, angularshaped nanoparticles were generated (Fig. S3A and $\mathrm{C}^{\dagger}$ ). In the case of $5 \mathrm{mmol}$ of $\mathrm{NaCl}$, only serious aggregation of A-SPIONs was observed (Fig. S3D †). After careful comparison, $1 \mathrm{mmol}$ of $\mathrm{NaCl}$ was chosen as the optimized condition to prepare the angular-shaped A-SPIONs in consideration of the shape and size uniformity (Fig. S3B $\dagger$ ).

Based on these results, we concluded that the combination of b-PEI and halide ions is highly related to the angular features of the synthesized A-SPIONs and chloride ions are more suitable compared to bromide ions. Thus, in further studies, we focused on A-SPIONs (9.43 $\pm 2.93 \mathrm{~nm}$, TEM observation) synthesized using $5 \mathrm{~g}$ PEG, $1 \mathrm{~g}$ b-PEI, and $1 \mathrm{mmol} \mathrm{NaCl}$. 

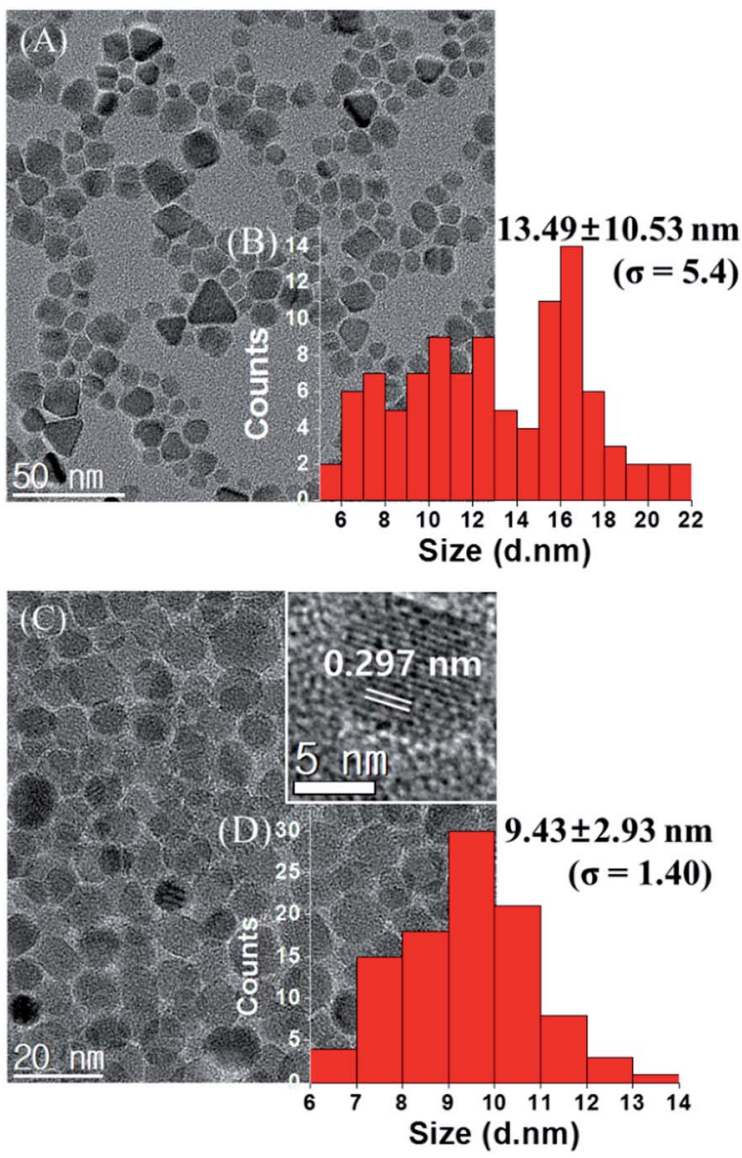

Fig. 2 TEM images of the A-SPIONs prepared with $1 \mathrm{mmol}$ of $\mathrm{KBr}(\mathrm{A})$ and $1 \mathrm{mmol}$ of $\mathrm{NaCl}(\mathrm{C})$. Size-distribution histograms of A-SPIONs with $1 \mathrm{mmol}$ of $\mathrm{KBr}(\mathrm{B})$ and $1 \mathrm{mmol}$ of $\mathrm{NaCl}(\mathrm{D})$. Lattice distances (d-spacing) of the synthesized nanocrystals were measured from the magnified HR-TEM images (inset).

The hydrodynamic size distribution of A-SPIONs was measured by DLS. The hydrodynamic size of the A-SPIONs dispersed in $0.1 \mathrm{M}$ saline was determined to be $13.97 \mathrm{~nm}$, as shown in Fig. 3. The DLS measurements and TEM images suggest that the A-SPIONs have a narrow size distribution and good water dispersibility.

\section{Crystal structure study of the A-SPIONs}

Regarding the crystal structure of the A-SPIONs, XRD measurement was carried out and the results are summarized in Fig. 4. The typical XRD patterns of the A-SPIONs show welldefined (200), (311), (222), (400), (422), (511), and (440) lines of $\mathrm{Fe}_{3} \mathrm{O}_{4}$ (JCPDS \# 00-019-0629). The (111) peak could not be assigned to PEG because it is overlapping the pattern of PEG, which is the surface organic molecule of A-SPIONs. ${ }^{42}$

The size of the crystalline region of the A-SPIONs was calculated using the Debye-Scherrer equation for the (220) diffraction peak $(K=0.9, \lambda=1.54 \AA)$. The calculated crystal size was found to be $10.02 \mathrm{~nm}$, which matches well with that obtained from the TEM images. The lattice adsorption band from the FT-IR spectra of A-SPIONs exhibits a peak at about $600 \mathrm{~cm}^{-1}$

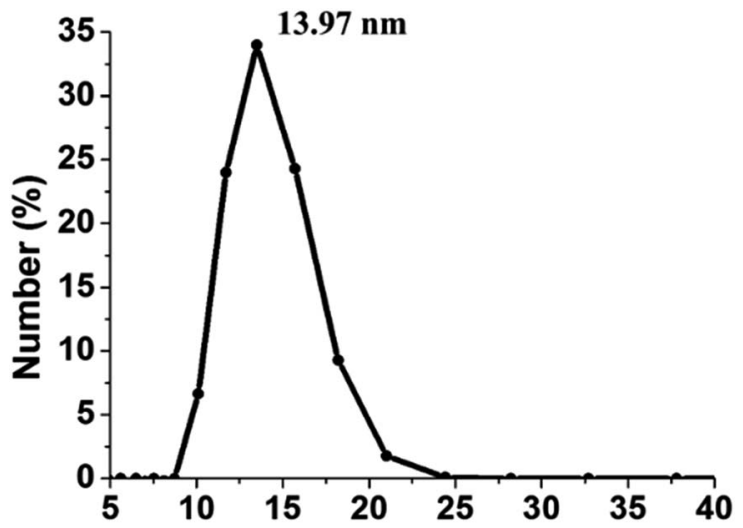

Fig. 3 Hydrodynamic size-distribution of the A-SPIONs in $0.1 \mathrm{M}$ saline.

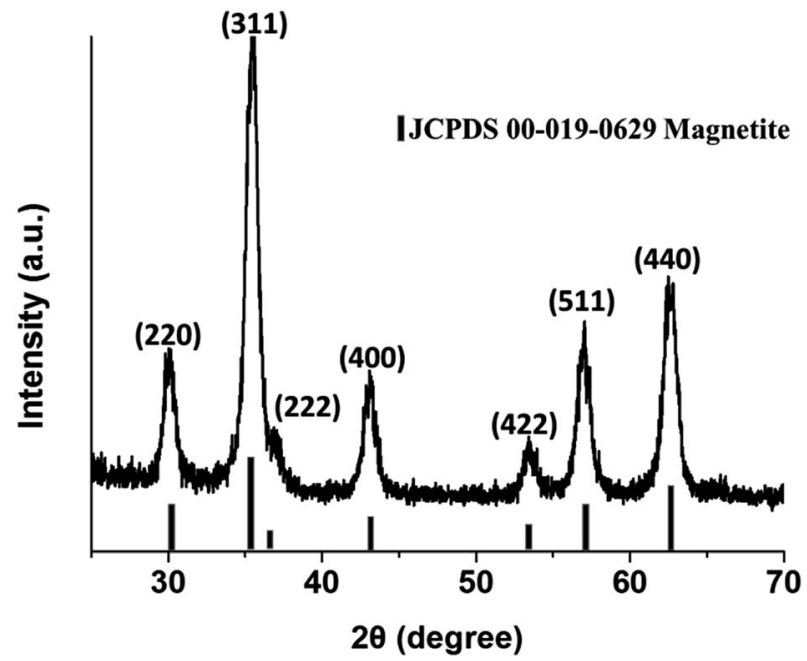

Fig. 4 XRD pattern of the synthesized A-SPIONs (5 g PEG, $1 \mathrm{~g}$ b-PEl, and $1 \mathrm{mmol} \mathrm{NaCl}$ ).

with a broad shoulder up to $750 \mathrm{~cm}^{-1}$, as shown in Fig. 5B, which indicates that the A-SPIONs are predominantly magnetite although they have a small oxide layer. ${ }^{43}$

\section{Surface studies of the A-SPIONs}

To quantify the amount of PEG and b-PEI on the surface of the A-SPIONs, we conducted TGA measurement from $25{ }^{\circ} \mathrm{C}$ to $1000{ }^{\circ} \mathrm{C}$ at the heating rate of $3{ }^{\circ} \mathrm{C} \mathrm{min}{ }^{-1}$ in air. About $3 \%$ weight loss was observed below $200{ }^{\circ} \mathrm{C}$ due to the evaporation of water from the A-SPIONs powder. After $200{ }^{\circ} \mathrm{C}$, thermal degradation of the surface capping polymers occurred, resulting in about 15 wt\% loss (Fig. 5A). Using ICP-MS analysis, the weight percentage of the surface capping polymers was calculated to be about $17.2 \mathrm{wt} \%$, which is nearly the same as the value obtained from the TGA analysis.

We also carried out FT-IR analysis to better understand the surface chemistry of the A-SPIONs, as shown in Fig. 5B. The characteristic peaks appeared at $3400 \mathrm{~cm}^{-1}, 2922 \mathrm{~cm}^{-1}, 2853$ $\mathrm{cm}^{-1}, 1615 \mathrm{~cm}^{-1}, 1431 \mathrm{~cm}^{-1}, 1381 \mathrm{~cm}^{-1}, 1286 \mathrm{~cm}^{-1}, 1110$ 

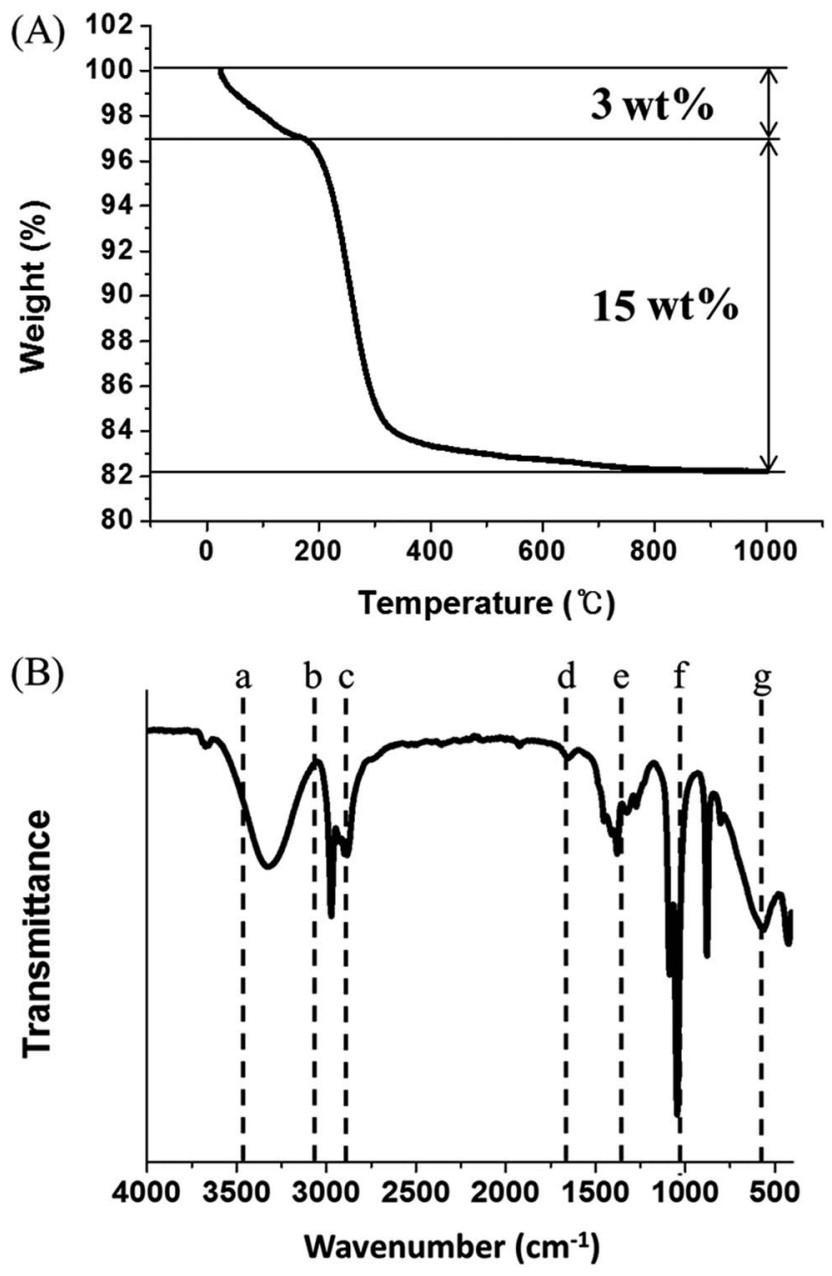

Fig. 5 TGA curve (A) and FT-IR spectra (B) of the as-synthesized ASPIONs. $a, b, c, d, e, f$ and $g$ in (B) are the characteristic peaks of the functional groups on the surface of A-SPIONs, a: $-\mathrm{OH}, \mathrm{b}:-\mathrm{NH}, \mathrm{c}$ : $-\mathrm{CH}_{2}, \mathrm{~d}$ and e: $-\mathrm{COO}$, f: $-\mathrm{CN}$, and g: $\mathrm{Fe}-\mathrm{O}$.

$\mathrm{cm}^{-1}$, and $585 \mathrm{~cm}^{-1}$. The broad band at $3400 \mathrm{~cm}^{-1}$ can be ascribed to the $\mathrm{O}-\mathrm{H}$ stretching vibration of PEG.

The peaks at about $1431 \mathrm{~cm}^{-1}, 2922 \mathrm{~cm}^{-1}$, and $2853 \mathrm{~cm}^{-1}$ are due to the $\mathrm{CH}_{2}$ groups of PEG and b-PEI. The peak at $1651 \mathrm{~cm}^{-1}$ can be ascribed to the asymmetric -COO stretching mode and the $-\mathrm{NH}_{2}$ bending mode, and the band at $1442 \mathrm{~cm}^{-1}$ can be assigned to the symmetric -COO stretching mode. The characteristic peak at $1286 \mathrm{~cm}^{-1}$ can be assigned to the $\mathrm{C}-\mathrm{N}$ bending vibration of b-PEI and the peak at about $580 \mathrm{~cm}^{-1}$ is due to the $\mathrm{Fe}-\mathrm{O}$ stretching vibration of the $\mathrm{Fe}_{3} \mathrm{O}_{4}$ nanoparticles. The FT-IR spectroscopic results confirmed that PEG and b-PEI were firmly attached onto the A-SPIONs surface, having amine and hydroxyl groups. Furthermore, due to the amine functional groups on the A-SPIONs surface, they exhibit the highly positive surface charge of $29.1 \mathrm{mV}$, which results in a stable dispersion in DI water (Fig. S4†).

\section{Magnetization study of the A-SPIONs}

The magnetic characteristic of the A-SPIONs was analyzed by drawing their $M-H$ loops in an applied field ranging from -10

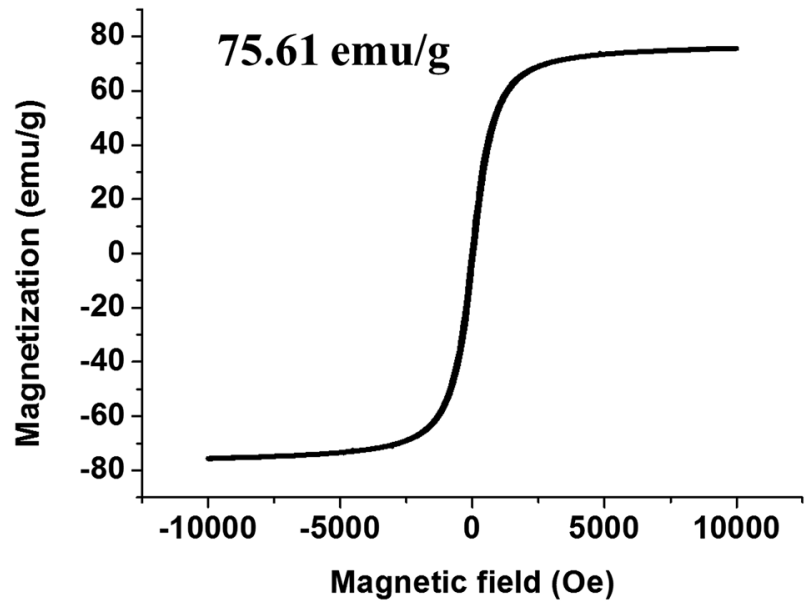

Fig. 6 SQUID magnetization measurement performed on the powder of $9.42 \pm 2.93 \mathrm{~nm}$ A-SPIONs.

kOe to 10 kOe at $298 \mathrm{~K}$ (Fig. 6). The $M-H$ curve of the A-SPIONs exhibits saturation magnetization $\left(M_{\mathrm{s}}\right)$ up to $75.61 \mathrm{emu} \mathrm{g}^{-1}$, and thus they have a superparamagnetic property. We assume that this high magnetization among iron oxide nanoparticles in a similar size range $\mathrm{e}^{\mathbf{1 4 4}}$ may be contributed by the angular shape of the nanocrystals.
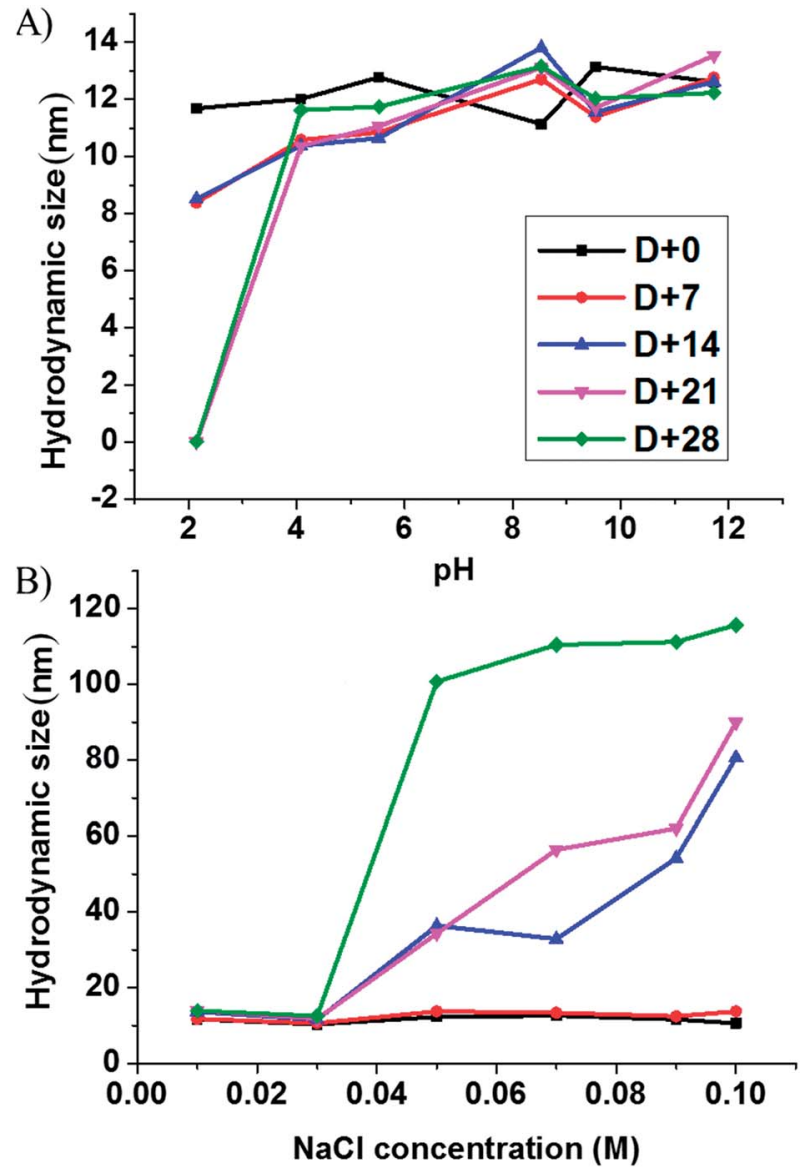

Fig. 7 Hydrodynamic size of the A-SPIONs dispersed in various $\mathrm{pH}(\mathrm{A})$ and $\mathrm{NaCl}$ concentrations for 28 days (B). 
There are several reports that compare magnetization according to the shape and size of the nanoparticles. Kovalenko et $a .^{45}$ and Zhen et al. ${ }^{32}$ reported that iron oxide nanocubes show superior saturation magnetization than spherical nanoparticles due to the shape-dependent effect. Recently, Zhou et $a{ }^{46}$ reported that the structural anisotropy of iron oxide nanoparticles is strongly related to their magnetic property and cubic-shaped iron oxide nanoparticles exhibit enhanced $r_{1}$ and $r_{2}$ (specific relaxivity) compared to spherical nanoparticles in MR imaging.

\section{Aqueous dispersion stability test of the A-SPIONs in various pH and $\mathrm{NaCl}$ concentrations}

Maintaining a stable dispersion is highly desirable for nanoparticles in the biomedical research area. In general, PEG has been widely used in the development of biomedical nanomaterials due to its biocompatibility and colloidal stability enhancement by the steric repulsive effect. The colloidal stability test of the as-synthesized A-SPIONs with an $\mathrm{Fe}$ concentration of $50 \mu \mathrm{g} \mathrm{mL} \mathrm{m}^{-1}$ was conducted in a broad range of solution $\mathrm{pH}$ (from $\mathrm{pH} 2.14$ to $\mathrm{pH} 11.73$ ) over a month. After 14 days, through DLS measurement (Fig. 7A) and data from the images (Fig. S5 $\dagger$ ), it was observed that the A-SPIONs in pH 2.14 completely dissolved; however, the hydrodynamic size under other $\mathrm{pH}$ conditions was maintained as that of their original colloidal states $(10-13 \mathrm{~nm})$.

A-SPIONs were also dispersed in solutions with various $\mathrm{NaCl}$ concentrations, ranging from $0.01 \mathrm{M}$ to $0.1 \mathrm{M}$, and were kept over a month. No precipitation was observed until 1 week; however, after 2 weeks, aggregation of A-SPIONs occurred at high concentration $(0.07-0.1 \mathrm{M})$. This is probably due to the strong electrostatic interaction between the surface of the nanoparticles and ions. Under $0.01 \mathrm{M}$ and $0.03 \mathrm{M} \mathrm{NaCl}$ conditions, no aggregation was observed for a month. These results confirm that the A-SPIONs have high dispersion stability over

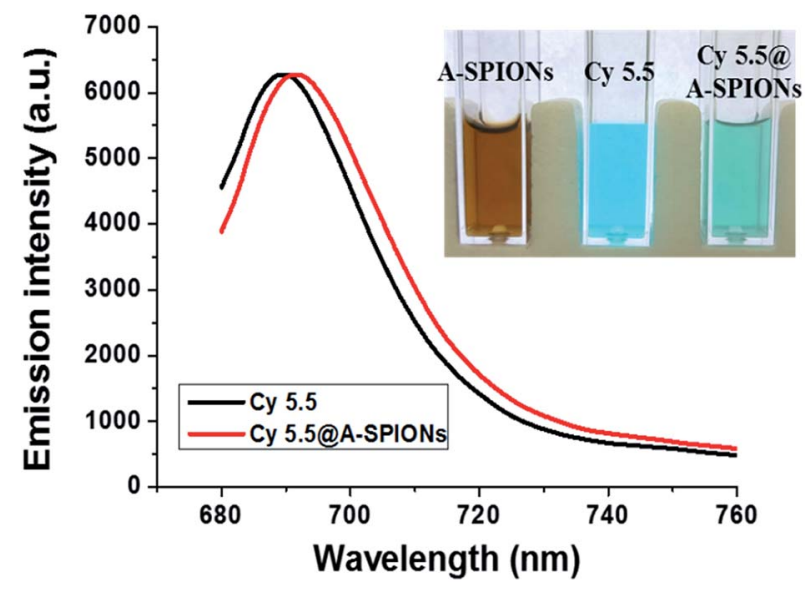

Fig. 8 Fluorescence spectra of Cy 5.5 and Cy 5.5@A-SPIONs. Cy 5.5 dye and Cy 5.5@A-SPIONs were excited at $675 \mathrm{~nm}$, and the emission was measured from 680 to $760 \mathrm{~nm}$ (inset: images of A-SPIONs, Cy 5.5 and Cy 5.5@A-SPIONs dispersed in distilled water). a broad range of $\mathrm{pH}$, whereas less stability in concentrated $\mathrm{NaCl}$ solutions.

\section{Fluorescence functionalization of the A-SPIONs}

The prepared A-SPIONs were labelled with Cyanine 5.5, one of the well-known near infrared (NIR) dyes commonly used for fluorescence imaging, by taking advantage of the amine-rich surface of the A-SPIONs. The Cy 5.5 dye-NHS ester was attached via amide-bond formation in a single step (Scheme 1).

The emission spectra of the free-standing Cy 5.5 and $\mathrm{Cy}$ 5.5@A-SPIONs are shown in Fig. 8. The free-standing Cy 5.5 dye shows maximum emission at $688.7 \mathrm{~nm}$ and Cy 5.5@A-SPIONs exhibits maximum intensity at $690.8 \mathrm{~nm}$, indicating that the fluorescence of the labelled Cy 5.5 dye was barely interfered by the iron oxide nanoparticles. We also tried to quantify the
(A)
U251
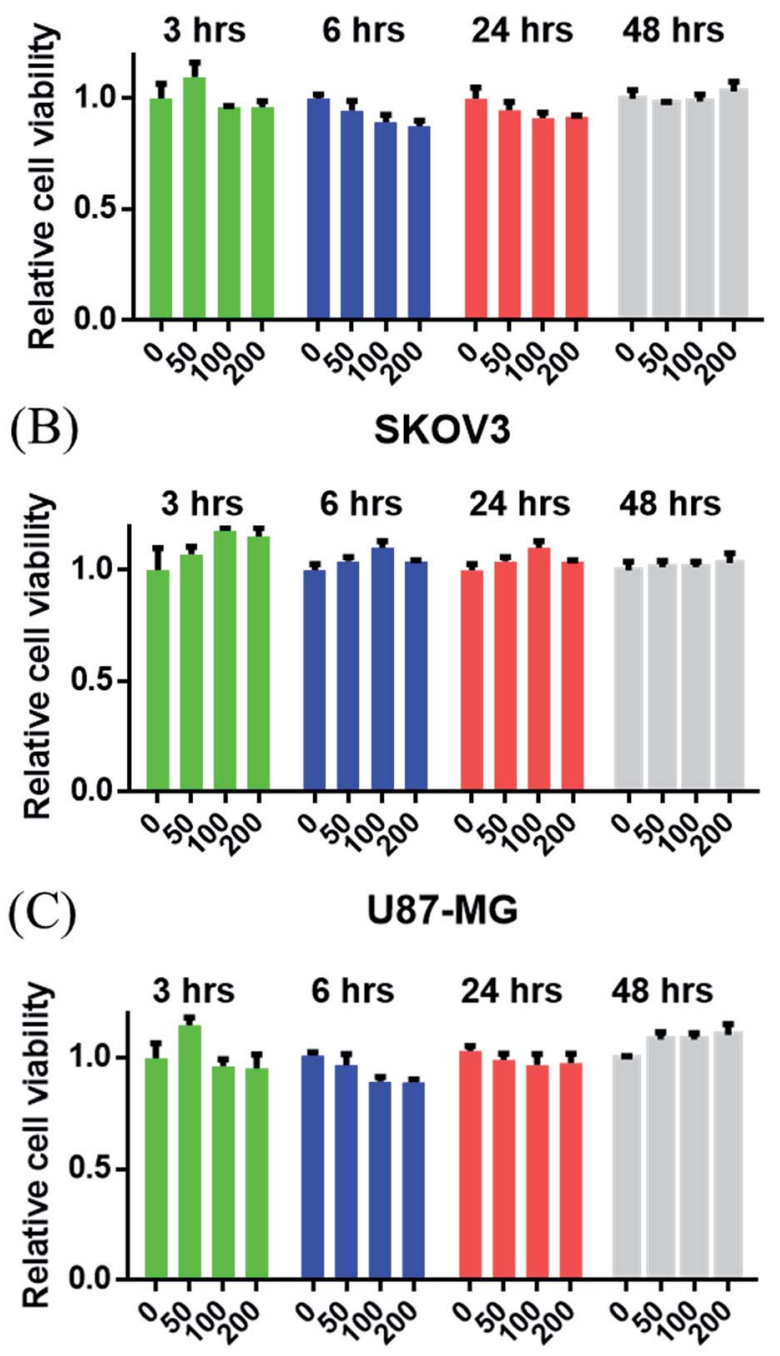

Fe ion concentration $(\mu \mathrm{g} / \mathrm{ml})$

Fig. 9 MTT assay results from three different types of cell lines (U251, SKOV3, and U87-MG) with an increase in the concentration of Fe ( $\mu \mathrm{g}$ $\mathrm{mL}^{-1}$ ) for 3, 6, 24, and 48 hours. 
attached Cy 5.5 dye on the surface of A-SPIONs by comparing the fluorescence intensity between the Cy 5.5 dye and Cy 5.5@ASPIONs. The standard curves of the free standing Cy 5.5 and Cy 5.5@A-SPIONs with various concentrations were achieved and are plotted in the ESI (Fig. S6 $\dagger$ ). The experimental results showed that a high loading of about $0.312 \mu \mathrm{mol}$ of Cy 5.5 dye was bound to $1 \mathrm{mg}$ of A-SPIONs.

\section{Cytotoxicity assessment}

Cellular viability was examined using the MTT assay method. Three types of cell lines were treated with three different concentrations of the nanoparticles and the results are shown in Fig. 9. More than $90 \%$ of the cells survived for 48 hours, regardless of the nanoparticle concentration up to $200 \mu \mathrm{g}$ Fe per $\mathrm{mL}$, proving that the as-prepared A-SPIONs have negligible cellular toxicity. This biological safety obtained via in vitro cytotoxic tests may guarantee their applications in the biomedical research area.

\section{In vitro fluorescent cell imaging}

The cellular uptake study of the Cy 5.5-labelled A-SPIONs was conducted using fluorescence confocal microscopy $(\times 60)$, as shown in Fig. 10. Nanoparticles in the fresh media ( $40 \mu \mathrm{g}$ Fe per $\mathrm{mL}$ ) were used to incubate RAW 264.7 cells for 12 hours. Blue and green fluorescence indicate the cell nucleus and plasma membrane, respectively, representing the overall morphology of the cells. The red signals, as shown in Fig. 10B, show the distribution of the Cy 5.5-labeled A-SPIONs absorbed by the cells. However, no red fluorescence signal was observed from the negative control experiment (Fig. 10A); thus, we concluded that the as-prepared nanoparticles were ingested enough and are highly acceptable as a fluorescent imaging nanoprobe for further in vivo studies.

\section{MR phantom imaging}

The principle of the $T_{2}$ MR contrast effect is that a magnetic dipole moment in superparamagnetic nanoparticles is induced under an applied magnetic field. When $\mathrm{H}_{2} \mathrm{O}$ molecules diffuse into the vicinity of the A-SPIONs with an induced dipole moment, the magnetic relaxation of the $\mathrm{H}_{2} \mathrm{O}$ protons is perturbed; thus, the $T_{2}$ (spin-spin relaxation time) is shortened. ${ }^{\mathbf{7}}$ Due to this effect, the signal decreases around the nanoparticles and a dark region on the $T_{2}$-weighted MR image appears. Fig. 11A shows the $T_{2}$-weighted MR phantom test from serially diluted iron oxide nanoparticles with various concentrations ([Fe]: $0.09-0.72 \mathrm{mM}$ ). The Fe concentration was determined from the ICP-MS data after dissolving in nitric acid solution. After the phantom imaging, we plotted the $R_{2}$ relaxation profile against Fe concentration. Due to the synthetic similarity of the previously reported USPIO ${ }^{19}$ and A-SPIONs, we prepared both in same Fe concentration to compare their $R_{2}$ relaxivity. Furthermore, we also compared Feridex®, which is commonly used as an MRI contrast agent for diagnosis. The calculated $R_{2}$ (specific relaxivity) of A-SPIONs is $215.37 \mathrm{mM}^{-1} \mathrm{~S}^{-1}$ (Fig. 11B), which is superior to that of USPIO $\left(51.19 \mathrm{mM}^{-1} \mathrm{~S}^{-1}\right)$ and Feridex® (152.92 $\mathrm{mM}^{-1} \mathrm{~S}^{-1}$ ). This difference probably originates from the saturation magnetization values. As shown in Fig. S1B, $\dagger$ the measured value of USPIO is $20.42 \mathrm{emu}^{-1}$, which is much smaller than that of the A-SPIONs (75.61 emu $\mathrm{g}^{-1}$ ).

Several factors such as structural composition, particle size ${ }^{47}$ and surface coating layer ${ }^{48}$ were widely investigated, associated with the relaxivity of iron oxide nanoparticles. $\mathrm{Hu}$ et al. ${ }^{48}$ reported that PEG-IONPs have an enhanced $r_{2}$ compared to DEG (diethylene glycol)-IONPs because PEG can carry more water molecules due to its longer polymer chains. This good performance of the A-SPIONs obtained via MR phantom test implies their potential for high-efficiency imaging in clinical diagnosis.

\section{Negative control (without A-SPIONs)}
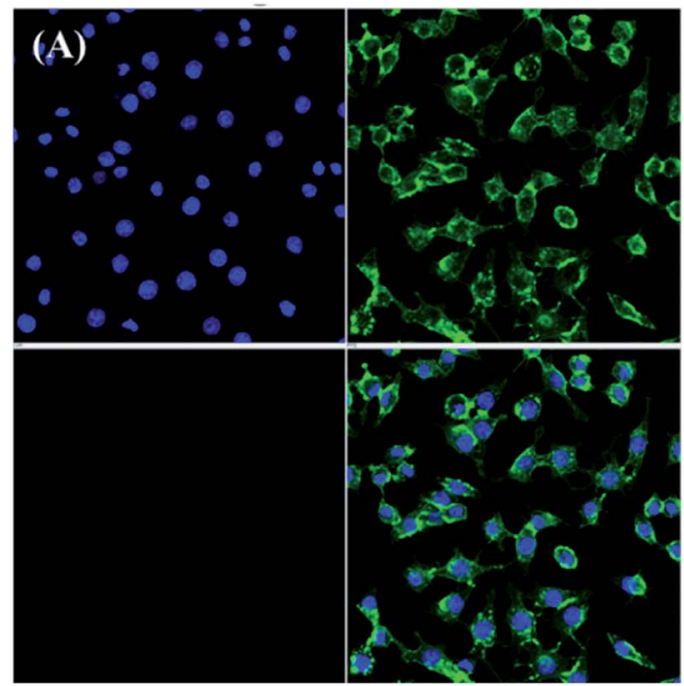

\section{$40 \mu \mathrm{g} \mathrm{Fe} / \mathrm{ml}$ of A-SPIONs for 12 hours}
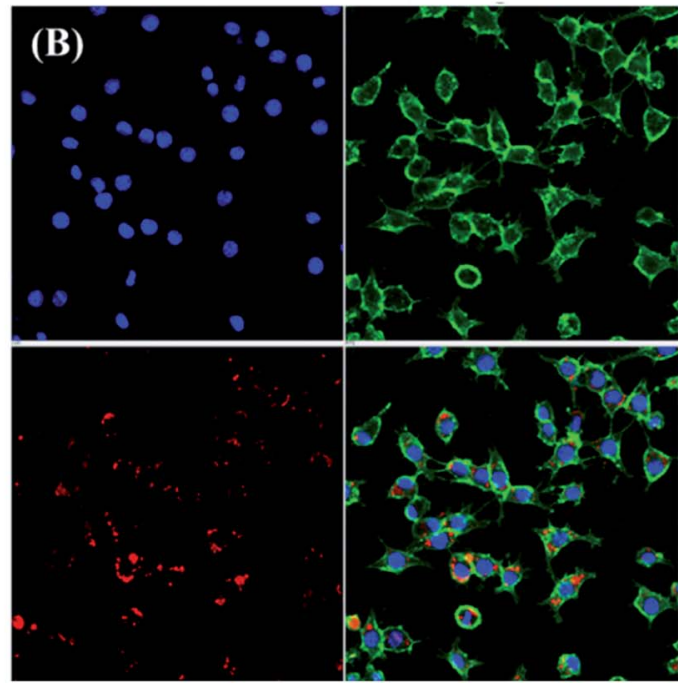

Fig. 10 Cellular uptake study of the Cy 5.5-labelled A-SPIONs via in vitro fluorescence imaging of RAW 264.7 cell lines ( $\times 60$ ). Cells were stained with Hoechst 33342 (cell nucleus, blue) and WGA-1 (plasma membrane, green). (A) Negative control and (B) incubated with $40 \mu \mathrm{g}$ Fe per mL for 12 hours. Red fluorescence from (B) shows strong cellular uptake of Cy 5.5-labelled A-SPIONs. 
(A) A-SPIONs USPIO Feridex ${ }^{\circledast}$

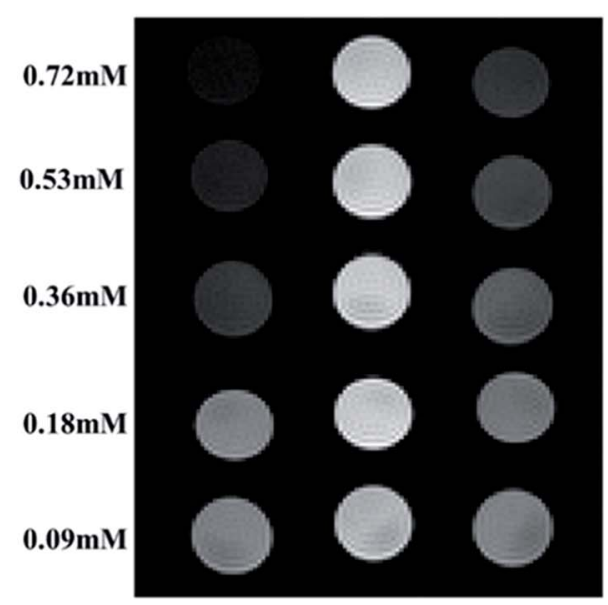

(B)

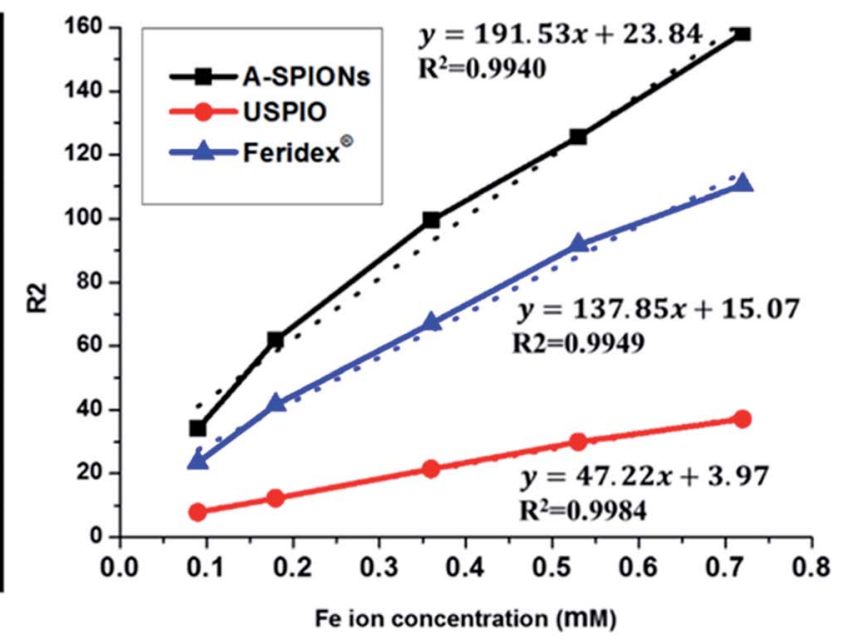

Fig. 11 (A) $T_{2}$-weighted MRI images of various concentrations of A-SPIONs (B) $R_{2}$ relaxation rates as a function of iron concentration (mM) of ASPIONs dispersed in DI water, measured at $25^{\circ} \mathrm{C}$ and $9.4 \mathrm{~T}$.

\section{Conclusions}

In this study, we introduced the robust synthesis of angularshaped amine-functionalized iron oxide nanoparticles using the polyol process with a mixture of PEG and b-PEI by collaborating with halide ions. The A-SPIONs exhibit stable dispersion in various aqueous media. The saturation magnetization value of $9.42 \pm 2.93 \mathrm{~nm}$ A-SPIONs was measured to be $75.61 \mathrm{emu} \mathrm{g}^{-1}$, which contributes to the superior enhancement of the $R_{2}$ relaxation of the nanoparticles compared to that of the previously reported iron oxide nanoparticles. The surface charge of A-SPIONs was measured to be $29.1 \mathrm{mV}$ due to their amine-rich surfaces; thus, NHS-functionalized Cy 5.5 dye labelling was easily accomplished via direct amide-bond formation. We also confirmed their cellular toxicity from SKOV3, U87MG, and U251 cell lines for up to 48 hours and the result was highly acceptable. Based on these results, we believe that the A-SPIONs will serve as a multipotent platform with high-efficiency $T_{2}$ contrast enhancement for MR imaging applications. In addition, the synthetic method may be applicable for the preparation of other functional nanomaterials for various biomedical applications.

\section{Acknowledgements}

This work was supported by NRF (National Research Foundation of Korea) Grant funded by the Korean Government (NRFNRF-2013H1A2A1033092-Fostering Core Leaders of the Future Basic Science Program/Global Ph.D. Fellowship Program) and Nano Material Technology Development Program (NRF2015M3A7B6027970) of MSIP/NRF. This work was also supported by the Center for Integrated Smart Sensors funded by the Ministry of Science, ICT and Future Planning, Republic of Korea, as Global Frontier Project (CISS-012M3A6A6054186). The authors would also like to thank Ga Ram Kim for in vitro cell imaging experiments.

\section{Notes and references}

1 R. Hao, R. Xing, Z. Xu, Y. Hou, S. Gao and S. Sun, Adv. Mater., 2010, 22, 2729-2742.

2 P. Sharma, S. Brown, G. Walter, S. Santra and B. Moudgil, Adv. Colloid Interface Sci., 2006, 123, 471-485.

3 Z. Hu, Y. Qu, K. Wang, X. Zhang, J. Zha, T. Song, C. Bao, H. Liu, Z. Wang and J. Wang, Nat. Commun., 2015, 6, 7560.

4 A. K. Gupta and M. Gupta, Biomaterials, 2005, 26, 3995-4021.

5 T. Neuberger, B. Schöpf, H. Hofmann, M. Hofmann and B. Von Rechenberg, J. Magn. Magn. Mater., 2005, 293, 483496.

6 J. Dobson, Drug Dev. Res., 2006, 67, 55-60.

7 C. J. Sunderland, M. Steiert, J. E. Talmadge, A. M. Derfus and S. E. Barry, Drug Dev. Res., 2006, 67, 70-93.

8 R. Massart, IEEE Trans. Magn., 1981, 17, 1247-1248.

9 I. Martínez-Mera, M. Espinosa-Pesqueira, R. PérezHernández and J. Arenas-Alatorre, Mater. Lett., 2007, 61, 4447-4451.

10 Y.-k. Sun, M. Ma, Y. Zhang and N. Gu, Colloids Surf., A, 2004, 245, 15-19.

11 J. Park, K. An, Y. Hwang, J.-G. Park, H.-J. Noh, J.-Y. Kim, J.-H. Park, N.-M. Hwang and T. Hyeon, Nat. Mater., 2004, 3, 891-895.

12 S. Sun, H. Zeng, D. B. Robinson, S. Raoux, P. M. Rice, S. X. Wang and G. Li, J. Am. Chem. Soc., 2004, 126, 273-279.

13 F. X. Redl, C. T. Black, G. C. Papaefthymiou, R. L. Sandstrom, M. Yin, H. Zeng, C. B. Murray and S. P. O'Brien, J. Am. Chem. Soc., 2004, 126, 14583-14599.

14 S. Ge, X. Shi, K. Sun, C. Li, C. Uher, J. R. Baker Jr, M. M. Banaszak Holl and B. G. Orr, J. Phys. Chem. C, 2009, 113, 13593-13599.

15 S. Takami, T. Sato, T. Mousavand, S. Ohara, M. Umetsu and T. Adschiri, Mater. Lett., 2007, 61, 4769-4772.

16 R. I. Walton, Chem. Soc. Rev., 2002, 31, 230-238. 
17 Y. Tian, B. Yu, X. Li and K. Li, J. Mater. Chem. B, 2011, 21, 2476-2481.

18 B. Zhang, Z. Tu, F. Zhao and J. Wang, Appl. Surf. Sci., 2013, 266, 375-379.

19 K. M. Yang, H.-I. Cho, H. J. Choi and Y. Piao, J. Mater. Chem. B, 2014, 2, 3355-3364.

20 W. Wu, Z. Wu, T. Yu, C. Jiang and W.-S. Kim, Sci. Technol. Adv. Mater., 2016, 16, 23501.

21 U. I. Tromsdorf, O. T. Bruns, S. C. Salmen, U. Beisiegel and H. Weller, Nano Lett., 2009, 9, 4434-4440.

22 B. Dubertret, P. Skourides, D. J. Norris, V. Noireaux, A. H. Brivanlou and A. Libchaber, Science, 2002, 298, 17591762.

23 H. B. Na, G. Palui, J. T. Rosenberg, X. Ji, S. C. Grant and H. Mattoussi, ACS Nano, 2011, 6, 389-399.

24 L. Diamandescu, D. Mihaila-Tarabasanu, N. PopescuPogrion, A. Totovina and I. Bibicu, Ceram. Int., 1999, 25, 689-692.

25 N. Li and W. H. Binder, J. Mater. Chem., 2011, 21, 1671716734.

26 J. Lu, M. Shi and M. S. Shoichet, Bioconjugate Chem., 2008, 20, 87-94.

27 R. A. Sperling and W. Parak, Philos. Trans. R. Soc., A, 2010, 368, 1333-1383.

28 H. Mattoussi, J. M. Mauro, E. R. Goldman, G. P. Anderson, V. C. Sundar, F. V. Mikulec and M. G. Bawendi, J. Am. Chem. Soc., 2000, 122, 12142-12150.

29 F. Dubois, B. Mahler, B. Dubertret, E. Doris and C. Mioskowski, J. Am. Chem. Soc., 2007, 129, 482-483.

30 J. H. Park, G. von Maltzahn, L. Zhang, M. P. Schwartz, E. Ruoslahti, S. N. Bhatia and M. J. Sailor, Adv. Mater., 2008, 20, 1630-1635.

31 P. Guardia, R. Di Corato, L. Lartigue, C. Wilhelm, A. Espinosa, M. Garcia-Hernandez, F. Gazeau, L. Manna and T. Pellegrino, ACS Nano, 2012, 6, 3080-3091.
32 G. Zhen, B. W. Muir, B. A. Moffat, P. Harbour, K. S. Murray, B. Moubaraki, K. Suzuki, I. Madsen, N. Agron-Olshina and

L. Waddington, J. Phys. Chem. C, 2010, 115, 327-334.

33 Z. Zhao, Z. Zhou, J. Bao, Z. Wang, J. Hu, X. Chi, K. Ni,

R. Wang, X. Chen and Z. Chen, Nat. Commun., 2013, 4, 2266. 34 Z. Xu, C. Shen, Y. Tian, X. Shi and H.-J. Gao, Nanoscale, 2010, 2, 1027-1032.

35 L. Feng, S. Zhang and Z. Liu, Nanoscale, 2011, 3, 1252-1257. 36 M. K. Yu, J. Park and S. Jon, Theranostics, 2012, 2, 3-44.

37 A. R. Vancha, S. Govindaraju, K. V. Parsa, M. Jasti, M. González-García and R. P. Ballestero, BMC Biotechnol., 2004, 4, 23.

38 The Polymer Data Handbook, ed. J. E. Mark, Oxford University Press, New York, 1999.

39 T. Yu, Z. Wu and W.-S. Kim, RSC Adv., 2014, 4, 37516-37521. 40 A. Shahzad, W.-S. Kim and T. Yu, RSC Adv., 2015, 5, 2865228661.

41 J. Cheon, N.-J. Kang, S.-M. Lee, J.-H. Lee, J.-H. Yoon and S. J. Oh, J. Am. Chem. Soc., 2004, 126, 1950-1951.

42 J. Lheritier, A. Chauvet and J. Masse, Thermochim. Acta, 1994, 241, 157-169.

43 T. Daou, J. Greneche, G. Pourroy, S. Buathong, A. Derory, C. Ulhaq-Bouillet, B. Donnio, D. Guillon and S. BeginColin, Chem. Mater., 2008, 20, 5869-5875.

44 K. Woo, J. Hong, S. Choi, H.-W. Lee, J.-P. Ahn, C. S. Kim and S. W. Lee, Chem. Mater., 2004, 16, 2814-2818.

45 M. V. Kovalenko, M. I. Bodnarchuk, R. T. Lechner, G. Hesser, F. Schäffler and W. Heiss, J. Am. Chem. Soc., 2007, 129, 63526353.

46 Z. Zhou, X. Zhu, D. Wu, Q. Chen, D. Huang, C. Sun, J. Xin, K. Ni and J. Gao, Chem. Mater., 2015, 27, 3505-3515.

47 Y. w. Jun, J. H. Lee and J. Cheon, Angew. Chem., Int. Ed., 2008, 47, 5122-5135.

$48 \mathrm{~F} . \mathrm{Hu}, \mathrm{K} . \mathrm{W}$. MacRenaris, E. A. Waters, T. Liang, E. A. Schultz-Sikma, A. L. Eckermann and T. J. Meade, J. Phys. Chem. C, 2009, 113, 20855-20860. 